\title{
Nonideal particle distributions from kinetic freeze-out models
}

\author{
Cs. Anderlik, ${ }^{1}$ Zs. I. Lázár, ${ }^{1}$ V. K. Magas, ${ }^{1}$ L. P. Csernai, ${ }^{1,2}$ H. Stöcker, ${ }^{3}$ and W. Greiner ${ }^{3}$ \\ ${ }^{1}$ Section for Theoretical Physics, Department of Physics, University of Bergen, Allegaten 55, 5007 Bergen, Norway \\ ${ }^{2}$ KFKI Research Institute for Particle and Nuclear Physics, P.O. Box 49, 1525 Budapest, Hungary \\ ${ }^{3}$ Institut für Theoretische Physik, Universität Frankfurt, Robert-Mayer-Strasse 8-10, D-60054 Frankfurt am Main, Germany
}

(Received 11 August 1998)

In fluid dynamical models the freeze-out of particles across a three-dimensional space-time hypersurface is discussed. The calculation of final momentum distribution of emitted particles is described for freeze-out surfaces, with both spacelike and timelike normals, taking into account conservation laws across the freeze-out discontinuity. [S0556-2813(99)01201-7]

PACS number(s): 25.75.-q, 25.75.Ld, 05.70.Ln

\section{INTRODUCTION}

The freeze-out of particle distributions is an essential part of continuum or fluid dynamical reaction models. From the point of view of observable consequences this is one of the most essential parts of the model. On the other hand this step is not based on fluid dynamical principles and governed by a large variaty of ad hoc assumptions. The freeze-out can be considered as a discontinuity across a hypersurface in spacetime.

The general theory of discontinuities in relativistic flow has not been worked out for a long time, and the 1948 work of Taub [1] discussed discontinuities across propagating hypersurfaces only (which have a spacelike normal vector $d \sigma^{\mu} d \sigma_{\mu}=-1$ ). Events happening on a propagating (twodimensional) surface belong to this category.

Another type of change in a continuum is an overall sudden change in finite volume. This is represented by a hypersurface with a timelike normal $d \sigma^{\mu} d \sigma_{\mu}=+1$ which is called, confusingly, both spacelike and timelike surfaces in the literature. In 1987 Taub's approach was generalized to both types of surfaces [2] making it possible to take into account conservation laws exactly across any surface of discontinuity in relativistic flow. This approach also eliminates the imaginary particle currents arising from the equation of the Rayleigh line. When the equation of state (EOS) is different on the two sides of the freeze-out front these conservation laws yield changing temperature, density and flow velocity across the front.

In fact the freeze-out surface is an idealization of a layer of finite thickness where the frozen-out particles are formed, and the interactions in the matter become gradually negligible. The dynamics of this layer can be described in different kinetic models or four-volume emission models [3]. The zero thickness limit of such a layer is the idealized freeze-out (FO) surface.

The invariant number of conserved particles (world lines) crossing a surface element $d \sigma^{\mu}$ is $d N=N^{\mu} d \sigma_{\mu}$ and the total number of all the particles crossing the FO hypersurface $S$ is $N=\int_{S} N^{\mu} d \sigma_{\mu}$. This total number $N$ and the total energy and momentum are of course the same at both sides of the freezeout surface. If we insert the kinetic definition of $N^{\mu}$

$$
N^{\mu}=\int \frac{d^{3} p}{p^{0}} p^{\mu} f_{\mathrm{FO}}\left(x, p ; T, n, u^{\nu}\right)
$$

into the integral over the freeze-out hypersurface $S$ we obtain the Cooper-Frye formula [4]

$$
E \frac{d N}{d^{3} p}=\int f_{\mathrm{FO}}\left(x, p ; T, n, u^{\nu}\right) p^{\mu} d \sigma_{\mu}
$$

where $f_{\mathrm{FO}}\left(x, p ; T, n, u^{\nu}\right)$ is the post $\mathrm{FO}$ phase space distribution of frozen-out particles which is not known from the fluid dynamical model. Problems usually arise from the bad choice of this distribution. First of all, to evaluate measurables we have to use the correct parameters of the matter after the FO discontinuity.

If we know the pre-freeze-out baryon current and energymomentum tensor $N_{0}^{\mu}$ and $T_{0}^{\mu \nu}$ we can calculate locally, across a surface element of normal vector $d \sigma^{\mu}$ the postfreeze-out quantities $N^{\mu}$ and $T^{\mu \nu}$, from the relations [1,2] $\left[N^{\mu} d \sigma_{\mu}\right]=0$ and $\left[T^{\mu \nu} d \sigma_{\mu}\right]=0$, where $[A] \equiv A-A_{0}$. In numerical calculations the local freeze-out surface can be determined most accurately via self-consistent iteration [7,9]. This fixes the parameters of our post FO momentum distribution $f_{\mathrm{FO}}\left(x, p ; T, n, u^{\nu}\right)$.

For example we can illustrate the effect of conservation laws for a situation where the frozen-out matter is massless baryon-free Bose gas. Then, the conservation laws across the freeze-out surface with timelike normal vector $d \sigma^{\mu}$ are $\left[T^{\mu \nu} d \sigma_{\nu}\right]=0$. In the most general (three-dimensional) case there are four parameters to be determined from the conservation laws: The final, post-FO temperature $T$, and three components of the velocity $u$. The energy-momentum tensor on the pre-freeze-out side, and the normal to the surface are given. The post-freeze-out energy-momentum tensor is of the form $T^{\mu \nu}=(e+p) u^{\mu} u^{\nu}-p g^{\mu \nu}$, where the energy density, pressure, and temperature are connected by the EOS: $e=\sigma_{\mathrm{SB}} T^{4}=3 p$, where $\sigma_{\mathrm{SB}}$ is the Stefan-Boltzmann constant. Then $T^{\mu \nu}=(e+p) u^{\mu} u^{\nu}-p g^{\mu \nu}$, can be written as a vector equation:

$$
\left(4 u^{\mu} u^{\nu} d \sigma_{\nu}-d \sigma^{\mu}\right)=x a^{\mu}
$$

where

$$
x=\left(\frac{1}{3} \sigma_{\mathrm{SB}} T^{4}\right)^{-1}, \quad a^{\mu}=T_{0}^{\mu \nu} d \sigma_{\nu} .
$$


Taking the normal projection of Eq. (2) and the norm of the four-velocity $u^{\mu}$, the solution for the four quantities we are looking for will be given by

$$
x=\frac{a^{\mu} d \sigma_{\mu}+\sqrt{\left(a^{\mu} d \sigma_{\mu}\right)^{2}+3 a^{\mu} a_{\mu}}}{a^{\mu} a_{\mu}}, \quad u^{\mu}=\frac{x a^{\mu}+d \sigma^{\mu}}{2 \sqrt{x a^{\mu} d \sigma_{\mu}+1}} .
$$

Idealized freeze-out across propagating discontinuities. One can go a step further in the study of the freeze-out process. We usually assume that the pre-freeze-out momentum distribution as well as the post-freeze-out distribution are both local thermal equilibrium distributions boosted by the local collective flow velocity on the actual side of the freezeout hypersurface, although, the post-freeze-out distribution need not be a thermal distribution

The case of freeze-out across a hypersurface with a spacelike normal shows this clearly because $p^{\mu}$ is timelike and $d \sigma^{\mu}$ is spacelike, thus $p^{\mu} d \sigma_{\mu}$ can be both positive and negative, i.e., $p^{\mu}$ may point now both in the post- and pre-FO directions. Thus the integrand in the above integral (1) may change sign in the integration domain, and this indicates that part of the distribution contributes to a current going back, into the front while another part is coming out of the front. On the pre-freeze-out side $p^{\mu}$ is unrestricted and $p^{\mu} d \sigma_{\mu}$ may really have both signs, because we may assume that the freeze-out front has a certain thickness [8], and due to internal rescatterings inside this front a current is fed back to the pre-freeze-out side to maintain the thermal equilibrium there.

On the post-freeze-out side, however, the distribution $f_{\mathrm{FO}}^{*}\left(x, p ; d \sigma^{\mu}\right)$ must vanish for momentum four-vectors $p^{\mu}$, which point back in the pre-FO direction, i.e., do not satisfy the condition $p^{\mu} d \sigma_{\mu}>0[6,7]$. Thus, this distribution cannot be a Jüttner or other ideal gas distribution. ${ }^{1}$

Nevertheless, the above conservation laws have to be satisfied even if the post-freeze-out distribution is not a local thermal distribution. Since the kinetic definitions of the energy-momentum tensor and conserved current(s) are reliably applicable, the conservation laws across a small element of the freeze-out front take the form

$$
\begin{gathered}
\int_{S}\left(\int \frac{d^{3} p}{p^{0}} f_{\mathrm{FO}}^{*}\left(x, p ; T, n, u^{\nu}, d \sigma^{\gamma}\right) p^{\mu}\right) d \sigma_{\mu}=\int_{S} N_{0}^{\mu}(x) d \sigma_{\mu} \\
\int_{S}\left(\int \frac{d^{3} p}{p^{0}} f_{\mathrm{FO}}^{*}\left(x, p ; T, n, u^{\sigma}, d \sigma^{\gamma}\right) p^{\mu} p^{\nu}\right) d \sigma_{\mu} \\
=\int_{S} T_{0}^{\mu \nu}(x) d \sigma_{\mu} .
\end{gathered}
$$

\footnotetext{
${ }^{1}$ Note that the contravariant normal when it becomes spacelike $d \sigma^{\mu}$ should point in the pre-FO direction to satisfy the condition $p^{\mu} d \sigma_{\mu}>0$, while the covariant normal $d \sigma_{\mu}$ always points in the post-FO direction. Thus, the direction of the contravariant normal $d \sigma^{\mu}$ in the Cooper-Frye formula goes continuously over from pointing in the pre-FO direction to pointing in the post-FO direction while the covariant normal of the FO surface stays directed always in the post-FO direction when it goes continuously over from timelike to spacelike.
}

Here, the matter is characterized by $T_{0}^{\mu \nu}$ and $N_{0}^{\mu}$ on the prefreeze-out side of the front.

The construction of the post-freeze-out distribution $f_{\mathrm{FO}}^{*}$ is a problem in the case of freeze out fronts with a spacelike normal. For the cut Jütner distribution the conserved currents were evaluated in Ref. [3]. Thus, if we know the five parameters of the pre-FO flow and the local freeze-out surface from kinetic considerations, then assuming that the post-FO distribution $f_{\mathrm{FO}}^{*}(p, x)$ is a cut Jütner distribution, we can completely determine the parameters of the post-FO matter from the conservation laws (4),(5). Although, this way we would formally satisfy the conservation laws and we would eliminate the particle current pointing back to the pre-FO matter, the strange shape of the cut Jütner distribution makes it difficult to accept it as a physical post-FO momentum distribution.

\section{FREEZE-OUT DISTRIBUTION FROM KINETIC THEORY}

Following the ideas introduced in Ref. [3] we can calculate the kinetic freeze-out distribution based on four-volume emission models. The proposed model, on the other hand, requires extended numerical calculation, so here we intend to study some overly simplified models, which might give us some hints about the expected shape of post-freeze-out distributions.

The freeze-out will turn out to be an exponential process, and after about three mean free paths the amount of interacting matter reduces to $5 \%$. Thus, the sharp freeze-out layer turns out to be an overidealization of kinetic freeze-out in heavy ion reactions, while it is applicable on more macroscopic scales such as in astrophysics. ${ }^{2}$

Let us first demonstrate the kinetic model for a drastically oversimplified situation of a plane FO surface. Let us assume an infinitely long tube with its left half $(x<0)$ filled with nuclear mater and in the right vacuum is maintained. We can remove the dividing wall at $t=0$, and then the matter will expand into the vacuum. By continuously removing particles at the right end of the tube and supplying particles on the left end, we can establish a stationary flow in the tube, where the particles will gradually freeze-out in an exponential rarefaction wave propagating to the left. We can move with this front, so that we describe it from the reference frame of the front (RFF).

In this frame, we have a stationary supply of equilibrated matter from the left, and a stationary rarefaction front on the right, $x>0$. We can describe the freeze-out kinetics on the right-hand side of the tube assuming that we have two components of our momentum distribution $f_{\text {free }}(x, \vec{p})$ and $f_{\text {int }}(x, \vec{p})$. However, we assume only that at $x=0 f_{\text {free }}$ vanishes exactly and $f_{\text {int }}$ is an ideal Jütner distribution (supplied

\footnotetext{
${ }^{2}$ On the other hand, if kinetic freeze-out coincides with a rapid phase transition, as in the case of rapid deconfinement transition of supercooled quark-gluon plasma, the short freeze-out hypersurface idealization may still be applicable even for heavy ion reactions. It is, however, beyond the scope of this work to study the freeze-out dynamics and kinetics in this latter case.
} 
by the inflow of equilibrated matter), while $f_{\text {int }}$ gradually disappears and $f_{\text {free }}$ gradually builds up as $x$ tends to infinity. We do not assume a priori that $f_{\text {int }}(x, \vec{p})$ is an ideal Jüttner distribution for all $x$, so we will have different FO results depending on the assumed FO mechanism.

Let us take first the most simple kinetic model describing the evolution of such a system. Starting from a fully equilibrated Jüttner distribution the two components of the momentum distribution develop according to the coupled differential equations

$$
\begin{aligned}
& \partial_{x} f_{\text {int }}(x, \vec{p}) d x=-\Theta\left(p^{\mu} d \sigma_{\mu}\right) \frac{\cos \theta_{\vec{p}}}{\lambda} f_{\text {int }}(x, \vec{p}) d x, \\
& \partial_{x} f_{\text {free }}(x, \vec{p}) d x=+\Theta\left(p^{\mu} d \sigma_{\mu}\right) \frac{\cos \theta_{\vec{p}}}{\lambda} f_{\text {int }}(x, \vec{p}) d x .
\end{aligned}
$$

Here the interacting component $f_{\text {int }}$ will deviate from the Jüttner shape and the solution will take the form

$$
f_{\text {int }}(x, \vec{p})=f_{\text {Jutner }}(x=0, \vec{p}) \exp \left[-\Theta\left(p^{\mu} d \sigma_{\mu}\right) \frac{\cos \theta_{\vec{p}}}{\lambda} x\right] .
$$

This solution is depleted in the forward $\vec{p}$ direction, particularly along the $x$ axis. Inserting it into the second differential equation above, leads to the freeze-out solution

$$
\begin{aligned}
f_{\text {free }}(x, \vec{p})= & f_{\text {Juttner }}(x=0, \vec{p}) \\
& \times\left\{1-\exp \left[-\Theta\left(p^{\mu} d \sigma_{\mu}\right) \frac{\cos \theta_{\vec{p}}}{\lambda} x\right]\right\} .
\end{aligned}
$$

At $x \rightarrow \infty$ this distribution will tend to the cut Jüttner distribution introduced in the previous section. (See Figs. 1-4.) The remainder of the original Jüttner distribution survives as $f_{\text {int }}$, even if $x \rightarrow \infty$. In this model the particle density does not change with $x$, barely particles moving faster than the freeze-out front (i.e., $p^{\mu} d \sigma_{\mu}>0$ ) are transferred gradually from component $f_{\text {int }}$ to component $f_{\text {free }}$. This is a highly unrealistic model, indicating that rescattering and rethermalization should be taken into account in $f_{\text {int }}$. This would allow particle transfer from the "negative momentum part" (i.e., $p^{\mu} d \sigma_{\mu}<0$ ) of $f_{\text {int }}$ to $f_{\text {free }}$, which is not possible otherwise.

\section{FREEZE-OUT DISTRIBUTION WITH RESCATTERING}

The assumption that the interacting part of the distribution remains the distorted (after some drain) Jüttner distribution, is of course highly unrealistic. Rescattering within this component will lead to rethermalization and reequilibration of this component. Thus the reequilibration and the drain terms are in competition and they mutually determine the evolution of the component $f_{\text {int }}$.

To include the collision terms explicitly into the transport equations (6) leads to a combined set of integrodifferential equations. We can, however, take advantage of the relaxation time approximation to simplify the description of the dynamics.

Then the two components of the momentum distribution

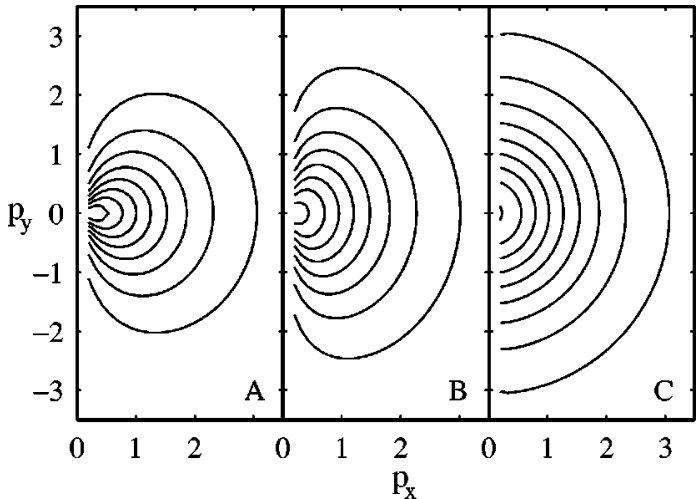

FIG. 1. The freeze-out distribution, $f_{\text {free }}(x, \vec{p})$, in the rest frame of the freeze-out front (RFF) calculated from the model presented in Sec. II. The momentum is plotted in units of $[T]$, and $T=m$ is assumed. Contour lines are given at values of $0.9,0.8,0.7, \ldots$, times the maximum of $f_{\text {free }}$. Here the center of the rest frame of the gas (RFG) is at rest in RFF, $u_{\mathrm{RFG}}^{\mu}=(1,0,0,0)$, however, the Eckart and Landau flow velocities of the frozen out matter do not vanish. (A), (B), (C) correspond to $x=0.02 \lambda, 3 \lambda, \infty$, respectively. At large distances from the initial point of the freeze-out process, $x$ $\rightarrow \infty(C)$, the distribution becomes a cut Jüttner distribution. The earlier stages of the freeze-out are, however, characterized by asymmetric distributions, elongated in the freeze-out direction $x$. This may lead to a large- $p_{t}$ enhancement, compared to the usual Jüttner assumption as freeze-out distribution used in most previous calculations.

develop according to the coupled differential equations

$$
\begin{aligned}
\partial_{x} f_{\mathrm{int}}(x, \vec{p}) d x= & -\Theta\left(p^{\mu} d \sigma_{\mu}\right) \frac{\cos \theta_{\vec{p}}}{\lambda} f_{\mathrm{int}}(x, \vec{p}) d x \\
& +\left[f_{\mathrm{eq}}(x, \vec{p})-f_{\mathrm{int}}(x, \vec{p})\right] \frac{1}{\lambda^{\prime}} d x, \\
\partial_{x} f_{\text {free }}(x, \vec{p}) d x= & +\Theta\left(p^{\mu} d \sigma_{\mu}\right) \frac{\cos \theta_{\vec{p}}}{\lambda} f_{\mathrm{int}}(x, \vec{p}) d x .
\end{aligned}
$$

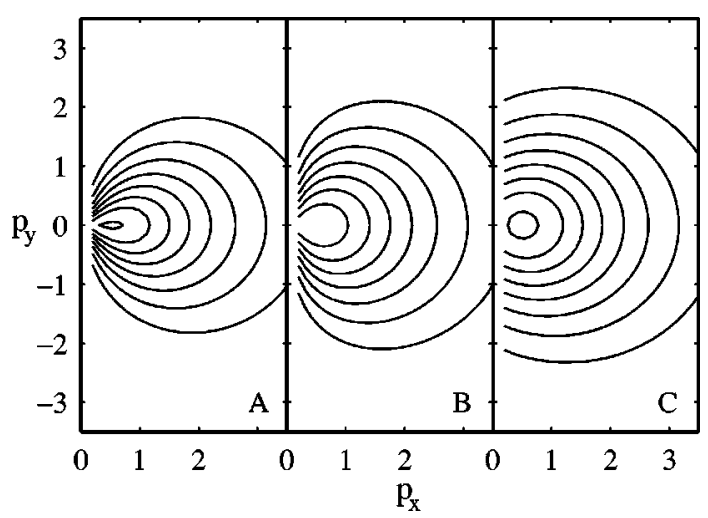

FIG. 2. The same as Fig. 1, except here the center of the rest frame of the gas is not at rest in RFF, $u_{\mathrm{RFG}}^{\mu}=(\gamma, 0.5,0,0)$. At large distances from the initial point of the freeze-out process, $x$ $\rightarrow \infty(C)$, the distribution becomes a cut Jüttner distribution, but less than half of the distribution is cut off. Note that the boosted Jüttner distribution became Lorentz elongated and asymmetric (see Fig. 2.10 of Ref. [5].) The earlier stages of the freeze-out, are characterized by asymmetric distributions, elongated in the freeze-out direction $x$. 


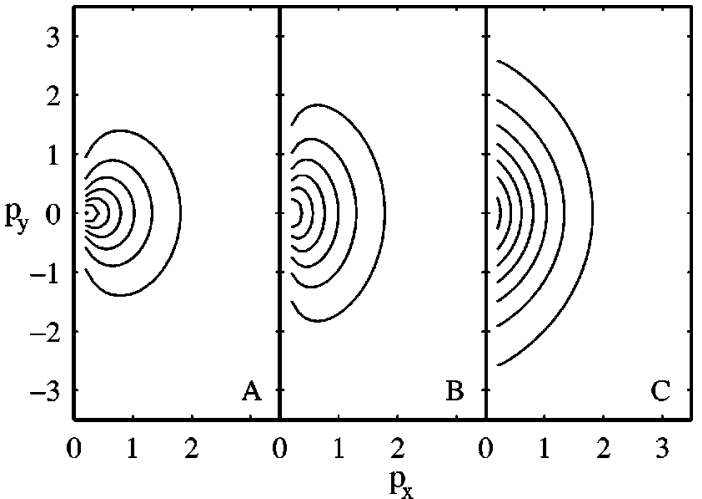

FIG. 3. The same as Fig. 1, except here the center of the rest frame of the gas is not at rest in RFF, $u_{\mathrm{RFG}}^{\mu}=(\gamma,-0.5,0,0)$. At large distances from the initial point of the freeze-out process, $x$ $\rightarrow \infty(C)$, the distribution becomes a cut Jüttner distribution, but more than half of the distribution is cut off. The earlier stages of the freeze-out, here also are characterized by asymmetric distributions, but these are not elongated in the freeze-out direction $x$.

Here, the interacting component of the momentum distribution shows the tendency to approach an equilibrated Jüttner type, distribution with a relaxation length coefficient $\lambda^{\prime}$ $\approx \lambda$. Of course due to the energy, momentum, and conserved particle drain, the distribution $f_{\text {eq }}(x, \vec{p})$ is not the same as the initial Jüttner distribution, but its parameters $n_{\mathrm{eq}}(x), T_{\mathrm{eq}}(x)$, and $u_{\mathrm{eq}}^{\mu}(x)$, change as required by the conservation laws.

Conservation laws. In this case the change of the conserved quantities caused by the particle transfer from component int to component free can be obtained in terms of the distribution functions as

$$
d N_{i}^{\mu}=-\frac{d x}{\lambda} \int \frac{d^{3} p}{p_{0}} p^{\mu} \Theta\left(p^{\mu} d \sigma_{\mu}\right) \cos \theta_{p} f_{\text {int }}(x, \vec{p})
$$

and

$$
d T_{i}^{\mu \nu}=-\frac{d x}{\lambda} \int \frac{d^{3} p}{p_{0}} p^{\mu} p^{\nu} \Theta\left(p^{\mu} d \sigma_{\mu}\right) \cos \theta_{p}^{*} f_{\text {int }}(x, \vec{p}) .
$$

If we do not have collision or relaxation terms in our transport equation then the conservation laws are trivially satisfied. If, however, collision or relaxation terms are present these contribute, to the change of $T^{\mu \nu}$ and $N^{\mu}$, and this should be considered in the modified distribution function $f_{\text {int }}(x, \vec{p})$.

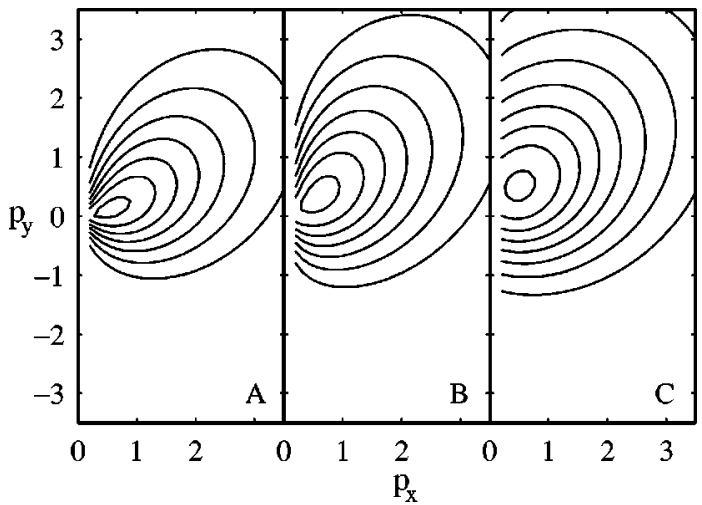

FIG. 4. The same as Fig. 1, except here the center of the rest frame of the gas is not at rest in RFF, $u_{\mathrm{RFG}}^{\mu}=(\gamma, 0.5,0.5,0)$. At large distances from the initial point of the freeze-out process, $x$ $\rightarrow \infty(C)$, the distribution becomes a cut Jüttner distribution, which is not centralized in $p_{y}$ and less than half of the distribution is cut off. The earlier stages of the freeze-out are characterized by distributions asymmetric both in the directions $p_{x}$ and $p_{y}$, and these are also elongated in the direction of the freeze-out flow velocity $u_{\mathrm{RFG}}^{\mu}$.

Immediate rethermalization limit. As a first approximation to the solution of Eqs. (9) let us assume that $\lambda^{\prime} \rightarrow 0$, i.e., we have immediate rethermalization after every step $d x$. Thus the drain is always happening from a component of shape $f_{\mathrm{eq}}(x, \vec{p})$, with parameters $\hat{n}(x), T(x)$, and $u_{\mathrm{RFG}}^{\mu}(x)$, and we can assume that $f_{\text {int }}=f_{\text {eq }}$ is of spherical Jüttner form at any $x$ including both positive and negative momentum parts. Above and henceforth the notation is similar to the one in Ref. [3]: $\tilde{n}=8 \pi T^{3} e^{\mu / T}(2 \pi \hbar)^{-3}, a=m / T$, so that $\hat{n}(\mu, T)=\tilde{n} a^{2} K_{2}(a) / 2$ is the invariant scalar density of the symmetric massless Jüttner gas, $b=a / \sqrt{1-v^{2}}, \quad v$ $=d \sigma_{0} / d \sigma_{x}, A=\left(2+2 b+b^{2}\right) e^{-b}$, and

$$
\mathcal{K}_{n}(z, w) \equiv \frac{2^{n}(n) !}{(2 n) !} z^{-n} \int_{w}^{\infty} d x\left(x^{2}-z^{2}\right)^{n-1 / 2} e^{-x},
$$

i.e., $\mathcal{K}_{n}(z, z)=K_{n}(z)$.

In this case the change of conserved quantities due to particle drain or transfer can be evaluated for an infinitesimal $d x$. We assume that the three-flow is normal to the freezeout surface, and for simplicity we assume $v>0$. In this case the change of the conserved particle currents in the RFF is given by

$$
\begin{aligned}
d N_{i}^{0}=-\frac{d x}{\lambda} & \frac{\tilde{n}}{4 v^{2} \gamma^{2}}\left\{b K_{1}(b)+b\left(3 v^{2}-1\right) \gamma^{2}\left[2 K_{1}(a)-\mathcal{K}_{1}(a, b)\right]+\gamma v^{2} b^{2}\left[2 K_{0}(a)-\mathcal{K}_{0}(a, b)\right]+2 v^{3} \gamma^{3}(b+1) e^{-b}\right\}, \\
d N_{i}^{x}= & -\frac{d x}{\lambda} \frac{\tilde{n}}{4 v^{3} \gamma^{3}}\left\{v^{2}\left(3 v^{2}-1\right) \gamma^{3} b\left[2 K_{1}(a)-\mathcal{K}_{1}(a, b)\right]+\left(2+v^{4} \gamma^{2} b^{2}\right)\left[2 K_{0}(a)-\mathcal{K}_{0}(a, b)\right]-2 K_{0}(b)\right. \\
& \left.+2 v \gamma^{2} e^{-b}\left[v^{2} \gamma^{2}(b+1)+v^{2} b-1\right]\right\},
\end{aligned}
$$

and for the change of the energy-momentum tensor in the RFF we obtain that 


$$
\begin{aligned}
d T_{i}^{00}= & -\frac{d x}{\lambda} \frac{\tilde{n} T}{4 v^{2} \gamma^{2}}\left\{v^{2} \gamma^{2} b^{2}\left(3+v^{2}\right)\left[2 K_{2}(a)-\mathcal{K}_{2}(a, b)\right]+\left(v^{2} b^{2}-v^{2}-1\right) \gamma b\left[2 K_{1}(a)-\mathcal{K}_{1}(a, b)\right]-b^{2}\left[2 K_{0}(a)-\mathcal{K}_{0}(a, b)\right]\right. \\
& \left.+\frac{a}{\gamma} K_{1}(b)+a^{2} K_{0}(b)+v \gamma^{2} e^{-b}\left[\left(1+3 v^{2}\right) \gamma^{2} A(b)-\left(2+v^{2} b^{2}\right)(b+1)+v^{4}\left(1+\frac{v^{2}}{3}\right) \gamma^{2} b^{3}\right]\right\} \\
d T_{i}^{0 x}= & -\frac{d x}{\lambda} \frac{\tilde{n} T}{4}\left\{\frac{1+3 v^{2}}{v} b^{2}\left[2 K_{2}(a)-\mathcal{K}_{2}(a, b)\right]+v a b^{2}\left[2 K_{1}(a)-\mathcal{K}_{1}(a, b)\right]+\left[v^{2} \gamma^{2} b\left(-a^{2}+\frac{1+3 v^{2}}{3 v} b^{2}\right)-b^{2}\right.\right. \\
& \left.\left.+\left(v^{2}+3\right) \gamma^{2} A(b)\right] e^{-b}\right\}-\frac{2 T}{v \gamma} d N_{i}^{0}, \\
d T_{i}^{x x}= & -\frac{d x}{\lambda} \frac{\gamma^{2} \tilde{n} T}{4 v}\left\{v\left(3+v^{2}\right) a^{2}\left[2 K_{2}(a)-\mathcal{K}_{2}(a, b)\right]+v^{3} a^{3}\left[2 K_{1}(a)-\mathcal{K}_{1}(a, b)\right]+\left[\frac{v^{4}}{3}\left(3+v^{2}\right) b^{3}+a^{2}\left(v^{4} b-1\right)\right.\right. \\
& \left.\left.+\left(3 v^{2}+1\right) A(b)\right] e^{-b}\right\}-\frac{3 T}{v \gamma} d N_{i}^{x}, \\
d T_{i}^{y y}=- & \frac{d x}{\lambda} \frac{\tilde{n} T}{8 v^{2} \gamma^{2}}\left[-\left(v^{2}+1\right) a\left[2 K_{1}(a)-\mathcal{K}_{1}(a, b)\right]-v^{2} a^{2}\left[2 K_{0}(a)-\mathcal{K}_{0}(a, b)\right]+\frac{a}{\gamma} K_{1}(b)-2 v(b+1) e^{-b}\right]+\frac{3 T}{2 v \gamma} d N_{i}^{x}
\end{aligned}
$$

and $d T_{i}^{z z}=d T_{i}^{y y}$. Note that in RFF the flow velocity of the rethermalized component is $u_{i, \mathrm{RFG}}^{\mu}(x)$ $=\left.\gamma_{\sigma}(x)(1, v(x), 0,0)\right|_{\mathrm{RFF}}$, where $\gamma_{\sigma}=1 / \sqrt{1-v^{2}}$.

The new parameters of distribution $f_{\text {int }}$, after moving to the right by $d x$ can be obtained from $d N_{i}^{\mu}$ and $d T_{i}^{\mu \nu}$. The conserved particle density of the rethermalized spherical Jüttner distribution after a step $d x$ is

$$
\hat{n}_{i}(x+d x)=\hat{n}_{i}(x)+d \hat{n}_{i}(x)=\sqrt{N_{i}^{\mu}(x+d x) N_{i, \mu}(x+d x)},
$$

where the expressions are invariant scalars. After straightforward calculation the differential equation describing the change of the proper particle density is

$$
d \hat{n}_{i}(x)=u_{i, \mathrm{RFG}}^{\mu}(x) d N_{i, \mu}(x) .
$$

Although this covariant equation is valid in any frame, we can calculate it in the RFF, where the values of $d N_{i}^{\mu}$ s were given above. Note again that the particle drain from $f_{\text {int }}(x)$, described by $d N_{i}^{\mu}$ is constrained to the "positive part" in the momentum space, but after rethermalization we attribute this to the change in the complete spherical Jüttner distribution $f_{\text {int }}(x+d x)$. Thus, in order to conserve momentum, we have to obtain a decreased Eckart flow velocity after the infinitesimal particle drain.

For the rethermalized interacting component Eckart's flow velocity is the velocity of the RFG, which changes with $x$, so we can actually denote this frame as $\operatorname{RFG}(x)$. For the spherical Jüttner distribution the Landau and Eckart flow ve- locities are the same, $u_{i, E, \mathrm{RFG}}^{\mu}(x)=u_{i, L, \mathrm{RFG}}^{\mu}(x)=u_{i, \mathrm{RFG}}^{\mu}(x)$. Thus we can evaluate the flow velocity $u_{i, \mathrm{RFG}}^{\mu}(x+d x)$

$$
u_{i, \mathrm{RFG}}^{\mu}(x+d x)=N_{i}^{\mu}(x+d x) / \sqrt{N_{i}^{\mu} N_{i, \mu}},
$$

which leads to the following covariant expression:

$$
d u_{i, E, \mathrm{RFG}}^{\mu}(x)=\Delta_{i}^{\mu \nu}(x) \frac{d N_{i, \nu}(x)}{\hat{n}_{i}(x)}
$$

where $\Delta_{i}^{\mu \nu}(x)=g^{\mu \nu}-u_{i, \mathrm{RFG}}^{\mu}(x) u_{i, \mathrm{RFG}}^{\nu}(x)$, is a projector to the plane orthogonal to $u_{i, \mathrm{RFG}}^{\mu}(x)$. This equation is valid in any reference frame, nevertheless we know the four-vectors on the right-hand side in the RFF explicitly. Then the new flow velocity of the matter evaluated according to Eckart's definition is $u_{i, E, \mathrm{RFG}}^{\mu}(x+d x)=u_{i, \mathrm{RFG}}^{\mu}(x)+d u_{i, E, \mathrm{RFG}}^{\mu}(x)$.

To get the temperature and the change of Landau's flow velocity, we have to analyze the change of the energy momentum tensor. Before the particle drain the energymomentum tensor at $x$ in the RFG is diagonal, $T_{i}^{\mu \nu}(x)$ $=\left.\operatorname{diag}\left(e_{i}, P_{i}, P_{i}, P_{i}\right)\right|_{\mathrm{RFG}(x)}$, while in the $\operatorname{RFF} T_{i}^{\mu \nu}(x)$ $=\left.\left[\left(e_{i}+P_{i}\right) u_{i, \mathrm{RFG}}^{\mu} u_{i, \mathrm{RFG}}^{\nu}(x)-P_{i} g^{\mu, \nu}\right]\right|_{\mathrm{RFF}(x)}$. Adding the drain terms $d T_{i}^{\mu \nu}(x)$ to this arising from the freeze-out while we move to the right by $d x$, yields $T_{i}^{\mu \nu}(x+d x)$ which will not be diagonal in the RFG $(x)$ and the pressure part will not be isotropic. We can Lorentz transform this to another frame which diagonalizes $T_{i}^{\mu \nu}(x+d x)$. This means to find the Landau flow velocity of the new system, $u_{i, L, \mathrm{RFG}}^{\mu}(x+d x)$ in the original $\operatorname{RFG}(x)$. After a straightforward diagonalization, 
somewhat tricky algebra, and neglecting second and higher order terms we arrive at the covariant expression ${ }^{3}$

$$
d u_{i, L, \mathrm{RFG}}^{\mu}(x)=\frac{\Delta_{i}^{\mu \nu}(x) d T_{i, \nu \sigma} u_{i, \mathrm{RFG}}^{\sigma}(x)}{e_{i}+P_{i}} .
$$

Although, for the spherical Jüttner distribution the Landau and Eckart flow velocities are the same, the change of this flow velocity when calculated from the baryon current and from the energy current are different:

$$
d u_{i, E, \mathrm{RFG}}^{\mu}(x) \neq d u_{i, L, \mathrm{RFG}}^{\mu}(x) .
$$

This is a clear consequence of the asymmetry caused by the freeze-out process as we pointed out already at the discussion of the properties of the cut Jütner distribution. Unfortunately, this also illustrates the weakness of our assumption on the complete rethermalization to a spherical Jüttner distribution, because we cannot choose the correct velocity change: If we choose $d u_{E}^{\mu}$ as the new velocity of the (spherical Jüttner distribution) $f_{\text {int }}(x+d x)$, then we violate the momentum conservation in our model, on the other hand if we choose $d u_{L}^{\mu}$, then we violate the baryon current conservation. Thus a spherical (or even elliptic) distribution cannot be fitted to the freeze-out drain, and we would have to use an ansatz which has (in addition) an asymmetry in the $x$ direction (i.e., an egg shape), for the distribution $f_{\text {int }}$.

Being aware of this weakness of the model, we nevertheless, maintain the assumption of spherical Jüttner shape for $f_{\text {int }}$ for the sake of simplicity. We can choose the flow velocity change then according to the physical problem. For example for the freeze-out of baryon free plasma this problem does not occur, and we have to choose $d u_{L}^{\mu}$.

The last item is to determine the change of the temperature parameter of $f_{\text {int }}$. From the relation $e \equiv u_{\mu} T^{\mu \nu} u_{\nu}$ we readily obtain the expression for the change of energy density

$$
d e_{i}(x)=u_{\mu, i, \mathrm{RFG}}(x) d T_{i}^{\mu \nu}(x) u_{\nu, i, \mathrm{RFG}}(x),
$$

and from the relation between the energy density and the temperature (see Chap. 3 in Ref. [5]), we can obtain the new temperature at $x+d x$. Fixing these parameters we fully determined the spherical Jüttner approximation for $f_{\text {int }}$. With

\footnotetext{
${ }^{3}$ Let the energy-momentum tensor of a system be $T^{\mu \nu}$. The energy and momentum flow is characterized by the Landau flow velocity, a unit four vector $u_{\mu}$. We are looking for a relationship between the infinitesimal change of the flow velocity $d u_{\mu}$ and the corresponding shift in the energy-momentum tensor $d T^{\mu \nu}$. We introduce the projector $\Delta^{\mu \nu}=g^{\mu \nu}-u^{\mu} u^{\nu}$ with the properties [5] $\Delta^{\mu \nu} u_{\nu}=0$ and $d u_{\mu}=\Delta_{\mu}^{\nu} d u_{\nu}$ since $u_{\mu} d u^{\mu}=0$. The Landau flow velocity is parallel to the flow of the momentum. Thus $u_{\mu}=$ const $\times T_{\mu}^{\nu} u_{\nu}$, therefore $\Delta_{\rho \mu} T^{\mu \nu} u_{\nu}=0$. We differentiate the above equation and take into consideration the identities $e \equiv u_{\mu} T^{\mu \nu} u_{\nu}$ and $\Delta_{\rho}^{\mu} T^{\rho \sigma} \Delta_{\sigma}^{\nu}=-P \Delta^{\mu \nu}$, where $e$ and $P$ are the energy density and pressure of the dissipationless, fully equilibrated fluid. Then using the properties of $\Delta^{\mu \nu}$ we get $d u_{\rho}(e+P)+u_{\rho} d u_{\mu} T^{\mu \nu} u_{\nu}=\Delta_{\rho \mu} d T^{\mu \nu} u_{\nu}$. Since the flow velocity and the momentum flow are parallel the second term on the left-hand side vanishes. Thus the equation describing the change of Landau's flow velocity becomes $d u_{\rho}=\Delta_{\rho \mu} d T^{\mu \nu} u_{\nu} /(e+P)$.
}

this ansatz the pressure asymmetry and pressure balance cannot be realized, thus our model will be only a rather approximate description of the freeze-out process. Nevertheless, we can draw some preliminary conclusions about the development of the kinetic distribution during freeze-out.

\section{CONCLUSIONS}

We turned to the problem of estimating the freeze-out distribution. Obviously the real freeze-out distribution depends strongly on the details of the freeze-out (and hadronization) dynamics. In heavy ion reactions, the curvature of the freeze-out surface and the conditions varying in time do affect the freeze-out distribution, nevertheless, as a first step, we assumed that the process is stationary and the curvature of the front is negligible. These approximations are extreme, but still enable us to draw some preliminary conclusions.

Following the lines and ideas presented in Ref. [3], the first simple kinetic freeze-out model reproduces the cut Jütner distribution as the limiting distribution $f_{\text {free }}$ after complete freeze-out at large distances. However, the model at the same time leads to unrealistic consequences, namely, that the interacting part of the distribution $f_{\text {int }}$ also survives fully, as the other part of the Jüttner distribution. Thus having both components at the end in this model, the physical freeze-out is actually not realized. This turns out to be a consequence of the fact that the effect of rescattering and thermalization in the interacting part of the distribution was ignored.

In an improved but still rather approximate kinetic freezeout model which takes rescatterings into account, the interacting component is assumed to be instantly rethermalized taking a spherical Jüttner shape at each time step with changing parameters. The model leads to a set of coupled differential equations (10)-(13). Equations (11) and (12) can be used in some combined form, or one of them can be selected which fits the physical situation the best. Then the three parameters of the interacting component $f_{\text {int }}$ can be obtained in each time step analytically [considering $\mathcal{K}_{n}(x, y)$ an analytic function].

Now the density of the interacting component will gradually decrease and disappear according to Eq. (10), the flow velocity will also decrease in both cases, Eqs. (11) or (12), because only forward going particles freeze-out, and the energy density will decrease also according to Eq. (13). Thus, the initial contribution to $f_{\text {free }}$ at small $x$ will resemble the distribution shown in Fig. 2(A), then as $x$ increases and the velocity decreases it will become to similar to Fig. 1(B), while at the final stages it will approach Fig. 3(C). As a consequence the integrated distribution will not resemble a cut Jüttner distribution.

Thus the arising post-freeze-out distribution $f_{\text {free }}$ will be a superposition of cut Jüttner type of components, from a series of gradually slowing down Jütner distributions. This will lead to a comet shaped final momentum distribution, with a more dominant leading head and a tail. In these rough models a large fraction $(\sim 95 \%)$ of the matter is frozen out by $x=3 \lambda$, thus the distribution $f_{\text {free }}$ at this distance can be considered as a first estimate of the post-freeze-out distri- 
bution. One should also keep in mind that the models presented here do not have realistic behavior in the limit $x$ $\rightarrow \infty$, due to their one-dimensional character. Nevertheless, this improved model with rescattering enables complete freeze out (unlike the simpler model in Sec. II where only the originally forward moving particles freeze-out even at large distances).

In case of rapid hadronization of QGP and simultaneous freeze out, the idealization of a freeze-out hypersurface may be justified, however, an accurate determination of the postfreeze-out hadron momentum distribution would require a nontrivial dynamical calculation.

\section{ACKNOWLEDGMENTS}

Enlightening discussions with F. Grassi, Y. Hama, and T. Kodama are gratefully acknowledged. This work was supported in part by the Research Council of Norway (programs for nuclear and particle physics, supercomputing, and free projects). Cs.A., L.P.C. and Zs.I.L. are thankful for the hospitality extended to them by the Institute for Theoretical Physics of the University of Frankfurt where part of this work was done. Cs.A. and Zs.I.L. are also indebted to the Theoretical Section of GSI, Darmstadt for their help.
[1] A.H. Taub, Phys. Rev. 74, 328 (1948).

[2] L.P. Csernai, Sov. Phys. JETP 65, 216 (1987); Zh. Eksp. Teor. Fiz. 92, 379 (1987).

[3] Cs. Anderlik, L.P. Csernai, F. Grassi, Y. Hama, T. Kodama, and Zs. Lázár, nucl-th/9806004.

[4] F. Cooper and G. Frye, Phys. Rev. D 10, 186 (1974).

[5] L.P. Csernai, Introduction to Relativistic Heavy Ion Collisions (Wiley, New York, 1994)
[6] Yu.M. Sinyukov, Sov. J. Nucl. Phys. 50, 228 (1989); Z. Phys. C 43, 401 (1989).

[7] K.A. Bugaev, Nucl. Phys. A606, 559 (1996).

[8] L.V. Bravina, I.N. Mishustin, N.S. Amelin, J.P. Bondorf, and L.P. Csernai, Phys. Lett. B 354, 196 (1995).

[9] J.J. Neumann, B. Lavrenchuk, and G. Fai, Heavy Ion Phys. 5, 27 (1997). 